\title{
Antiquantization of the Double Confluent Heun Equation. The Teukolsky Equation
}

\author{
A. A. Salatich, S. Yu. Slavyanov
}

Different forms of the double confluent Heun equation are studied. A generalized Riemann scheme for these forms is given. An equivalent first-order system is introduced. This system can be regarded from the viewpoint of the monodromy property. A corresponding Painleve equation is derived by means of the antiquantization procedure. It turns out to be a particular case of $P^{3}$. A general expression for any Painlevé equation is predicted. A particular case of the Teukolsky equation in the theory of black holes is examined. This case is related to the boundary between spherical and thyroidal geometries of a black hole. Difficulties for its antiquantization are shown.

Keywords: double confluent Heun equation, antiquantization, Painlevé equation $P^{3}$, Teukolsky equation

\section{Introduction}

The idea of antiquantization as a tool of generating Painlevé equations from Heun equations had been proposed by one of the authors of this paper in the publication [1]. Further, the presentation was extended and corrected (unfortunately with several typos) in the book [2]. However, there still remain some gaps where considerations were purely heuristic. These gaps were partly covered in the recent publications $[3,4]$. In view of the fact that only the basic Heun equation was studied there in detail additional particular computations for confluent and other particular cases of Heun equations are needed. Below we just put one brick in this direction.

The double-confluent Heun equation may be the most mysterious in this set. On the other hand, it is applied to many physical problems: in optics, hydrodynamics and gravity. Here we give different forms of this equation, the corresponding first-order linear system with the isomonodromic property, we choose the normalization of the accessory parameter and derive the corresponding Painlevé equation which turns out to be a specific $P^{3}$.

Received December 16, 2018

Accepted February 13, 2019

\author{
Alexander A. Salatich \\ salatichaa@mail.ru \\ Sergey Yu. Slavyanov \\ s.slavyanov@spbu.ru \\ Saint-Petersburg State University \\ Universitetskaya nab. 7/9, Saint-Petersburg, 199034 Russia
}


As the most fascinating example the particular Teukolsky equation for the specific geometry of the boundary surface of the black hole is examined.

\section{The double confluent Heun equation}

The double-confluent Heun equation (DHE) reads [2]

$$
(H(z, D)-t h) w(z):=\left(z^{2} D^{2}+\left(-z^{2}+c z-t\right) D+(-a z-t h)\right) w(z)=0, \quad D=d / d z
$$

Its corresponding generalized Riemann scheme reflecting the asymptotic behavior at irregular singularities $z=0$ and $z=\infty$ is [2]

$$
\left(\begin{array}{ccc}
2 & 2 & \\
0 & \infty & ; z \\
0 & a & ; h \\
2-c & c-a & \\
0 & 0 \\
t & 1
\end{array}\right) .
$$

Here $c, a$ are parameters related to the above-mentioned singularities, $t$ is a varying parameter and $h$ is a nonlocal parameter known as the accessory parameter. The latter parameter is normalized by factor $\mathrm{t}$ in front of it. Our goal is to justify the choice of normalization for needs of antiquantization [3] and to connect the equation under study to the linear first-order system of Schlesinger type. Below we use the following notations:

$$
\sigma(z)=z^{2}, \quad \tau(z)=-z^{2}+c z-t, \quad \omega(z)=-a z,
$$

which simplify the intermediate calculations.

There is a broad field of applications of DHE in physics, for instance, in the Kerr solution of the Einstein equation [5] in some hydrodynamics problems etc.

We start with

Lemma 1. With the chosen normalization of the accessory parameter $h$ the DHE reduces at $t=0$ to a confluent hypergeometric equation.

Proof. Let $t=0$ in (2.1) and divide it by the common factor $z$. Then we obtain

$$
z w^{\prime \prime}(z)+w^{\prime}(z)(c-z)-a w(z)=0
$$

which turns out to be a confluent hypergeometric equation.

Equation (2.1) is known as DHE in canonical form. In addition to this form the self-adjoint form is often considered, namely,

$$
\left(H_{s a}(z, D)-t h\right) u(z):=\left(D z^{2} D-\left(\frac{z}{2}-\frac{t}{2 z}\right)^{2}-\left(a-\frac{c}{2}\right) z-\frac{t(c / 2-1)}{z}-t h\right) u(z)=0
$$

with relation to the canonical form as

$$
y(z)=z^{1-c / 2} e^{z / 2+t /(2 z)} u(z) .
$$


Equation (2.3) simplifies to

$$
D \sigma(z) D u(z)+Q(z) u(z)-t \lambda u(z)=0 .
$$

The corresponding generalized Riemann scheme is

$$
\left(\begin{array}{ccc}
2 & 2 & \\
0 & \infty & ; z \\
-1+c / 2 & a+1-c / 2 & ; h \\
1-c / 2 & 1+c / 2-a & \\
-t / 2 & -1 / 2 \\
t / 2 & 1 / 2
\end{array}\right) .
$$

\section{Linear $2 \times 2$ first-order system}

Here we follow the recipe proposed for the Heun equation in [4]. This recipe explains the antiquantization procedure to the isomonodromic property.

Consider a linear $2 \times 2$ first-order system

$$
R \overrightarrow{Y^{\prime}}=\left(\begin{array}{cc}
z-\rho & z \\
-\rho & z
\end{array}\right) \overrightarrow{Y^{\prime}}=A \vec{Y}=\left(\begin{array}{cc}
z & e_{2} \\
e_{3} & e_{4}
\end{array}\right) \vec{Y},
$$

with $\operatorname{det} A=e_{4} z-e_{2} e_{3}$ and $\operatorname{det} R=z^{2}$. Note that $\operatorname{det} R=0$ at $z=0$. The matrix $R(z)$ can be regarded as a polynomial rotation.

It can be transformed to the system

$$
z^{2} \vec{Y}^{\prime}=T \vec{Y}
$$

where

$$
T(z)=\left(\begin{array}{cc}
z & -z \\
\rho & z-\rho
\end{array}\right) A=\left(\begin{array}{cc}
z\left(z-e_{3}\right) & z\left(e_{2}-e_{4}\right) \\
\rho z-(\rho-z) e_{3} & \rho e_{2}-(\rho-z) e_{4}
\end{array}\right) .
$$

The linear second-order equation for the first component $y_{1}(z)$ can be derived as follows:

$$
z^{2} y_{1}^{\prime \prime}+y_{1}^{\prime}\left(c z-z^{2}-t\right)-y_{1}(a z+t h)=0,
$$

which coincides with DHE (2.1) under the conditions

$$
\begin{aligned}
c z-z^{2}-t & =-\operatorname{tr} T-z^{2}\left(\ln \frac{T_{12}}{z^{2}}\right)^{\prime}=-z^{2}+z\left(e_{3}-e_{4}+1\right)+\rho\left(e_{4}-e_{2}\right)-a z-t h= \\
& =\operatorname{det} A+T_{12}\left(\frac{T_{11}}{T_{12}}\right)^{\prime}=z\left(e_{4}+1\right)-e_{2} e_{3} .
\end{aligned}
$$

These equalities lead to the following relations between the parameters of DHE and the parameters of the system:

$$
\begin{aligned}
t & =-\rho\left(e_{4}-e_{2}\right), \\
c & =e_{3}-e_{4}+1, \\
a & =-\left(e_{4}+1\right), \\
h & =-\frac{e_{2} e_{3}}{\rho\left(e_{4}-e_{2}\right)} .
\end{aligned}
$$


Being inverted these equalities give

$$
\begin{gathered}
e_{4}=-(a+1), \quad e_{3}=c-a-2, \quad e_{2}=\frac{t h}{c-a-2}, \\
\rho=\frac{t}{\frac{t h}{c-a-2}+a+1}, \\
T(z)=\left(\begin{array}{cc}
z\left(z-c^{\prime}\right) & z\left(\frac{t h}{c^{\prime}}+a+1\right) \\
z c^{\prime}+\left(\begin{array}{c}
t \\
\frac{t h}{c^{\prime}}+a+1
\end{array}\right)\left(z-c^{\prime}\right) & z(-a-1)+t
\end{array}\right)
\end{gathered}
$$

where $c^{\prime}=c-a-2$.

The matrix formulation of the double confluent Heun equation enables one to find different symmetries typical of its solutions [6]. Moreover, the isomonodromic condition for it gives a Painlevé equation.

Two types of linear first-order system are studied in relation to Heun class equations. Matrices for the first type have zero determinants at singularities, matrices for the second type have zero trace at singularities. Here the first case is considered.

\section{Antiquantization for DHE}

According to the general antiquantization procedure (see, for instance, [4]), the Hamiltonian for classical movement with variables $q, p$ ( $q$ being the coordinate and $p$ the momentum) in the case of canonical DHE is

$$
H(q, p)=\frac{1}{t}\left(\sigma(q) p^{2}+\tau(q) p+\omega(q)\right) .
$$

Turning to velocity $\dot{q}$ according to

$$
\dot{q}=\frac{\partial H}{\partial p}=\frac{2 p \sigma(q)+\tau(q)}{t}
$$

the Lagrangian $\mathcal{L}(q, \dot{q})$ can be obtained as

$$
\mathcal{L}=\dot{q} p-H=\frac{t}{4 \sigma(q)}(\dot{q}-\tau(q) / t)^{2}-\frac{\omega(q)}{t} .
$$

Hence, the Euler - Lagrange equation reads

$$
\frac{d}{d t}\left(\frac{t \dot{q}}{q}\right)=\frac{q^{2}+q(2 a-c)}{t}+\frac{c-1}{q}-\frac{t}{q^{2}}
$$

This equation is a particular (however, genuine!!!) case of the equation

$$
\ddot{q}-\frac{\dot{q}^{2}}{q}+\frac{\dot{q}}{t}-\gamma q^{3}-\frac{\alpha q^{2}+\beta}{t}-\frac{\delta}{q}=0,
$$

which is known as the Painlevé $P^{3}$ equation in general form. The proof of it is obtained by substitution for new variables $t^{:}=t^{2}, q:=t q$, which does not violate the integrability of the equation. Existence of a larger number of parameters in (4.4) in comparison with (4.2) is explained by the fact that (4.4) includes also results of antiquantization for two reduced DHE's. 
In the case of a self-adjoint form of DHE (2.4) we also arrive at $P^{3}$, which could be predicted.

We formulate a general conjecture related to the link between the Heun class equations and the Painlevé equations.

Conjecture. Every Heun class equation in a canonical form can be written as

$$
\sigma(z, t) y^{\prime \prime}(z, t)+\tau(z, t) y^{\prime}(z, t)+\omega(z, t) y(z, t)=f(t) h y(z, t)
$$

where $\sigma(z), \tau(z), \omega(z)$ are polynomials of degree not higher than three and the normalization factor $f(t)$ chosen from the condition that at its zeros equation (4.5) simplifies to an equation of hypergeometric class.

Then, after the antiquantization procedure, Eq. (4.5) generates the Painlevé equation in the following general form:

$$
\ddot{q}-\frac{\dot{q}^{2}}{2} \frac{d \ln (\sigma(q))}{d q}+\dot{q}\left(\frac{\partial \ln (\sigma(q))}{\partial t}-\frac{d \ln (f(t))}{d t}\right)=F(q, t),
$$

where $F(q, t)$ is a rational function of its arguments.

In the case of the Heun equation this conjecture has been proved in [7].

\section{The Teukolsky equation in gravitation theory}

In the Kerr model of gravity equations for black holes the Teukolsky equations [5, 8-11] are often studied. Omitting the angular equations, we turn to the radial equation which under certain assumptions (namely, in the case of the so-called extremal model) can be presented as a particular DHE. Firstly, consider the following quantity:

$$
\Delta=r^{2}-2 M r+a^{2},
$$

where $r$ is the radius for the observation point, $M$ is the mass of the black hole and $a$ is a specific parameter characterizing the geometrical shape of a black hole. For $a<M$ the ergosphere (exterior domain) of the black hole is topologically equivalent to a sphere and for $a>M$ it is topologically equivalent to a torus. We are studying the boundary case $a=M$ below (Fig. 1). Other cases are investigated in a series of papers of other authors. The Teukolsky equation in the general case for radial function $R_{l m}(r)$ reads

$$
\Delta \frac{d^{2} R_{l m}}{d r^{2}}+2(s+1)(r-M) \frac{d R_{l m}}{d r}+V(r) R_{l m}=0,
$$

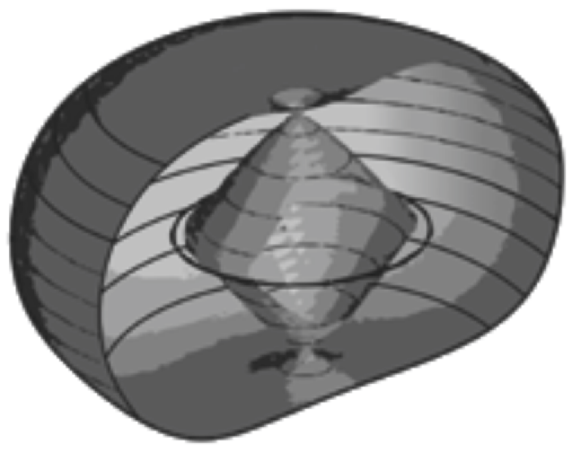

Fig. 1. A plot of the ergo surface for the extremal case: $a=\mathrm{M}[9]$. 
with

$$
V(r)=-A_{l m}+\frac{\omega^{2} r^{2}\left(r^{2}+a^{2}\right)+2 \omega M r\left(\omega a^{2}+2 a m\right)+m^{2} a^{2}}{\Delta} .
$$

Here $s$ is a parameter denoted as spin. We set $s=0$ for simplicity. Index $m$ which takes nonnegative integral values arises from the angle equation along the $\phi$ variable. Index $l$ enumerates eigenvalues $A_{l m}$ with respect to the angular equation along the $\theta$ variable. Taking the particular units of mass, we also set $M=1 / 2$.

Then, when $a=1 / 2$, we arrive at a simplified Teukolsky equation

$$
z^{2} \frac{d^{2} R_{l 0}}{d z^{2}}+2 z \frac{d R_{l 0}}{d z}+Q(z) R_{l 0}=0
$$

with

$$
Q(z)=\omega^{2} z^{2}+2 \omega^{2} z+\frac{\omega^{2}}{z}+\frac{1}{4} \frac{\omega^{2}}{z^{2}}+\frac{11}{2} \omega^{2}-A_{l 0} .
$$

In order to compare (5.3) with DHE in self-adjoint form, the following scaling transform is needed:

$$
\omega z=\zeta / 2, \quad \omega^{2}=t / 2,
$$

which does not change the differential part of (5.3) but leads to

$$
Q(\zeta)=\zeta^{2} / 4+\sqrt{t / 2} \zeta+\frac{t \sqrt{t / 2}}{\zeta}+\frac{t^{2}}{4 \zeta^{2}}-t h
$$

The relation between the Teukolsky equation and the self-adjoint form of DHE is established. However, the parameters $c$ and $a$ turn out to be dependent on parameter $t$, namely, $c / 2-a=$ $=\sqrt{t / 2}, 1-c / 2=\sqrt{t / 2} \rightarrow a=1-\sqrt{2 t}, c=2-\sqrt{2 t}$. That makes the antiquantization procedure ambiguous. Further studies on this issue are needed. An algorithm for calculation of the abovementioned eigenfunctions can be found in [12]. To conclude, we would like to mention the paper [13], where Painlevé transcendents are directly used for scattering off black holes.

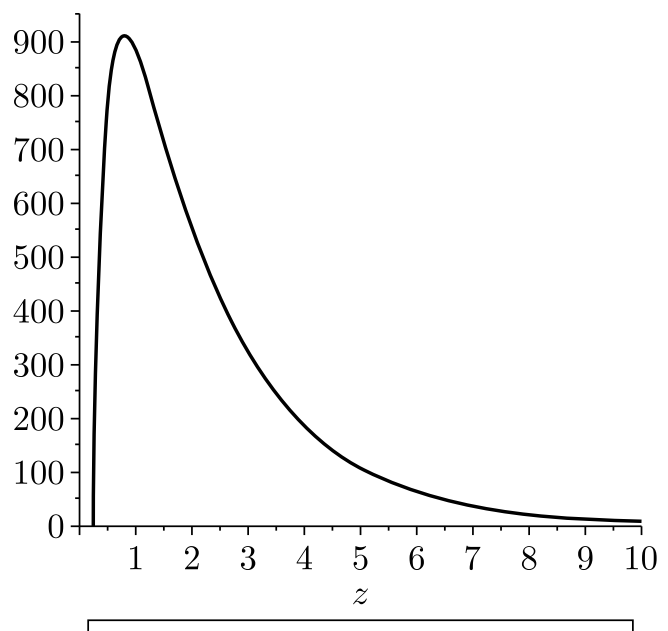

Doubleconfluent Heun function

Fig. 2. A plot of the eigenfunction of the Teukolsky equation for $t=1, a=1-\sqrt{2}, c=2-\sqrt{2}$. 


\section{Conclusion}

The summary of the results obtained in the article is:

1. A lemma for normalization of the accessory parameter is proved.

2. A first-order system equivalent to DHE is derived.

3. The general form of the Painlevé equations is formulated.

4. The Teukolsky equation for the particular geometry of the Kerr model is presented in terms of the self-adjoint form of DHE.

The preliminary version of the paper has been presented as a talk at the Russian-Chinese conference on integrable systems and geometry, EIMI, Saint-Petersburg, Russia. The authors are thankful to referees for valuable comments.

\section{References}

[1] Slavyanov, S. Yu., Painlevé Equations As Classical Analogues of Heun Equations, J. Phys. A, 1996, vol. 29, no. 22, pp. 7329-7335.

[2] Slavyanov, S. Yu. and Lay, W., Special Functions: A Unified Theory Based on Singularities, Oxford: Oxford Univ. Press, 2000.

[3] Slavyanov, S. Yu. and Stesik, O. L., Antiquantization of Deformed Heun-Class Equations, Theoret. and Math. Phys., 2016, vol. 186, no.1, pp.118-125; see also: Teoret. Mat. Fiz., 2016, vol. 186, no.1, pp. $142-151$.

[4] Babich, M. and Slavyanov, S., Antiquantization, Isomonodromy, and Integrability, J. Math. Phys., 2018, vol. 59, no. 9, 091416, 11 pp.

[5] Staicova, D. and Fiziev, P., The Spectrum of Electromagnetic Jets from Kerr Black Holes and Naked Singularities in the Teukolsky Perturbation Theory, in Trends in Particle Physics (Primorsko, Bulgaria, 2010).

[6] Kazakov, A. Ya. and Slavyanov, S. Yu., Euler Integral Symmetries for the Confluent Heun Equation and Symmetries of the Painlevé Equation PV, Theoret. and Math. Phys., 2014, vol.179, no.2, pp. 543-549; see also: Teoret. Mat. Fiz., 2014, vol.179, no. 2, pp. 189-195.

[7] Slavyanov, S. Yu., Kovalevskaya's Dynamics and Schrödinger Equations of Heun Class, in Operator Methods in Ordinary and Partial Differential Equations (Stockholm, 2000), S. Albeverio, N. Elander, W. N. Everitt, P. Kurasov (Eds.), Oper. Theory Adv. Appl., vol. 132, Basel: Birkhäuser, 2002, pp. 395-402.

[8] Teukolsky, A. S., Rotating Black Holes: Separable Wave Equations for Gravitational and Electromagnetic Perturbations, Phys. Rev. Lett., 1972, vol. 29, no. 16, pp. 1114-1118.

[9] Staicova, D. and Fiziev, P., The Spectrum of Electromagnetic Jets from Kerr Black Holes and Naked Singularities in the Teukolsky Perturbation Theory, Astrophys. Space Sci., 2011, vol.332, no. 2, pp. 385-401.

[10] Casals, M. and Micchi, L.F. L., Spectroscopy of Extremal (and Near-Extremal) Kerr Black Holes, arXiv:1901.04586v2 (2019).

[11] London, L. and Fauchon-Jones, E., On Modeling for Kerr Black Holes: Basis Learning, QNM Frequencies, and Spherical-Spheroidal Mixing Coefficients, arXiv:1810.03550v2 (2019).

[12] Lay, W., Bay, K., and Slavyanov, S. Yu., Asymptotic and Numeric Study of Eigenvalues of the Double Confluent Heun Equation, J. Phys. A, 1998, vol. 31, no. 42, pp. 8521-8531.

[13] Novaes, F. and de Cunha, B. C., Isomonodromy, Painlevé Transcendents and Scattering off of Black Holes, arXiv:1404.5188 (2014). 